\title{
Screening masses in strong external magnetic fields
}

\author{
Claudio Bonat:* \\ Dipartimento di Fisica e Astronomia dell'Università di Firenze and INFN - Sezione di Firenze, \\ Via Sansone 1, 50019, Sesto Fiorentino (FI), Italy.

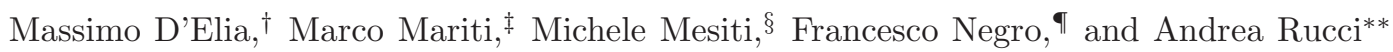 \\ Dipartimento di Fisica dell'Università di Pisa and INFN - Sezione di Pisa, \\ Largo Pontecorvo 3, I-56127 Pisa, Italy. \\ Francesco Sanfilippd \\ INFN, Sezione di Roma Tre, \\ Via della Vasca Navale 84, I-00146 Rome, Italy.
}

(Dated: April 28, 2017)

\begin{abstract}
We present results for the (color)magnetic and (color)electric screening masses of the Quark-Gluon Plasma in the presence of an external magnetic field. The screening masses are extracted from the correlators of Polyakov loops, determined by lattice QCD simulations at the physical point. We explore temperatures in the range $200 \mathrm{MeV} \lesssim T \lesssim 330 \mathrm{MeV}$ and magnetic field intensities up to $|e| B \sim 1.3 \mathrm{GeV}^{2}$. We find that both screening masses are increasing functions of the magnetic field and that the dependence on $B$ becomes weaker for larger temperatures. In the case of the magnetic screening mass a slight anisotropy is also observable.

PACS numbers: 12.38.Gc, 12.38.Mh, 12.38.Aw
\end{abstract}

\section{INTRODUCTION}

The fate of heavy quark bound states as a probe of the deconfining properties of the thermal strongly interacting medium has a long history. In the seminal paper Ref. [1] a suppression of the production rate of these states was predicted, being caused by the shortening of the screening length for color interactions in the QuarkGluon Plasma. In subsequent analyses it was realized that the situation is in fact more involved: various effects can enhance or suppress this phenomenon, the final result being the outcome of a complex dynamical process (see Ref. 2] for a recent review of the theoretical and experimental aspects). Here we consider the consequences that the introduction of a magnetic background field may have on the screening lengths of the thermal medium.

The presence of strong magnetic backgrounds, with field strengths comparable to the QCD scale, is a situation common to many contexts, ranging from cosmology [3, 4] and non-central heavy ion collisions [5-10], with magnetic fields going up to $10^{16}$ Tesla $\left(e B \sim 1 \mathrm{GeV}^{2}\right)$, to magnetars [11], where magnetic fields of the order of $10^{11}$ Tesla are expected on the surface, and possibly higher in the inner core.

\footnotetext{
*Electronic address: claudio.bonati@unifi.it

$\dagger$ Electronic address: massimo.delia@unipi.it

${ }^{\ddagger}$ Electronic address: mariti@df.unipi.it

$\S$ Electronic address: michele.mesiti@pi.infn.it

IElectronic address: fnegro@pi.infn.it

**Electronic address: andrea.rucci@pi.infn.it

${ }^{\dagger \dagger}$ Electronic address: francesco.sanfilippo@roma3.infn.it
}

How the properties of strongly interacting matter are modified by such magnetic fields has been the subject of many theoretical efforts, see Refs. [12, 13] for recent reviews. As regards the effects more directly related to color interactions, various studies have considered the possible influence of an external magnetic field on the static quark-antiquark potential 14 19], which has been clarified by recent lattice results [20, 21], and might have consequences relevant to the spectrum of heavy quark bound states 22 32]. At zero temperature, the potential becomes anisotropic and the string tension $\sigma$ is larger (smaller) in the direction orthogonal (parallel) to the magnetic field B [20, 21]; at finite $T$, in particular in the region right below the pseudocritical temperature $T_{c}$, the magnetic field induces a general suppression of $\sigma$ [21], leading to an early onset of deconfinement, in agreement with the observed dependence of $T_{c}$ on $B$ [33 33 , 35$]$

In this paper we extend the study to the region of temperatures above $T_{c}$, in order to investigate the effects of a magnetic background on the interactions between heavy quarks in the Quark-Gluon Plasma. In this phase, the effective interaction is no longer confining and can instead be described by a screened Coulomb form, with two different screening lengths/masses characterizing the (color)electric and the (color)magnetic sectors. Studying the appropriate combinations of Polyakov loop correlators by means of Lattice QCD simulations at the physical point we estimated the screening lengths for $T \simeq 200,250,330 \mathrm{MeV}$ and several values of the external magnetic field (for $|e| B \lesssim 1.3 \mathrm{GeV}^{2}$ ). From these results we conclude that the magnetic field induces a reduction of both screening lengths (i.e. an increasing of the screening masses), which might induce a further sup- 
pression of the formation of heavy quark bound states in the thermal medium.

The paper is organized as follows. In Section II we discuss our numerical setup and review the definition of the screening masses in terms of Polyakov loop correlators. In Section III we present our numerical results. Finally, in Section IV, we draw our conclusions.

\section{SETUP}

\section{A. Physical observables}

The screening masses of strongly interacting matter have been historically introduced in perturbation theory by studying the pole structure of the finite temperature gluon propagator. While this approach presents no difficulties for the computation of the leading order electric screening mass (see e.g. [36 38]), it was soon realized that perturbation theory gets into trouble at higher orders, or even at the leading order for the magnetic mass, because of the infrared sensitivity of the obtained expressions $37-42]$.

The natural way to overcome these difficulties and obtain nonperturbative results for the screening masses is to analyze the large distance behavior of gauge-invariant correlation functions. For this purpose, correlators of Polyakov loops have been traditionally used [43, 44] and the two independent correlators that can be studied are

$$
\begin{aligned}
& C_{L L^{\dagger}}(\mathbf{r}, T)=\left\langle\operatorname{Tr} L(\mathbf{0}) \operatorname{Tr} L^{\dagger}(\mathbf{r})\right\rangle \\
& C_{L L}(\mathbf{r}, T)=\langle\operatorname{Tr} L(\mathbf{0}) \operatorname{Tr} L(\mathbf{r})\rangle,
\end{aligned}
$$

where $L(\mathbf{x})$ is the Polyakov loop operator, which is defined in the continuum by

$$
L(\mathbf{x})=\frac{1}{N_{c}} \mathcal{P} \exp \left(-i g \int_{0}^{1 / T} A_{0}(\mathbf{x}, \tau) \mathrm{d} \tau\right) .
$$

In this expression $N_{c}$ is the number of colors and the symbol $\mathcal{P} \exp$ denotes the path-ordered exponential.

The correlator $C_{L L^{\dagger}}$ is often studied because of its connection with the free energy $F_{Q \bar{Q}}(\mathbf{r}, T)$ of a static quarkantiquark pair, that can be computed using the relation [45]

$$
F_{Q \bar{Q}}(\mathbf{r}, T)=-T \log C_{L L^{\dagger}}(\mathbf{r}, T) .
$$

The study of $C_{L L^{\dagger}}$ is thus the finite temperature counterpart of the study of Wilson loops at zero temperature, from which the potential energy of a static quarkantiquark pair can be extracted. Since in any numerical study the temporal extent of the lattice is always finite, it would also be possible to extract the static potential as the zero temperature limit of the free energy extracted from Polyakov loops, however this second procedure is generically not numerically convenient because of the much larger statistical errors involved.

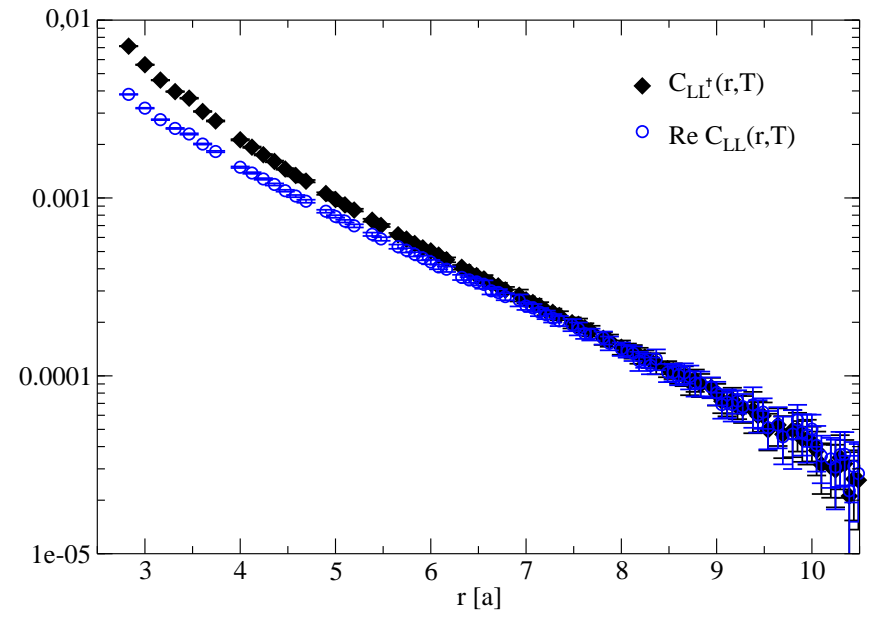

FIG. 1: Comparison of the correlator $C_{L_{L \dagger}}(r, T)$ and the real part of the correlator $C_{L L}(r, T)$ for $T \simeq 200 \mathrm{MeV}$ (in both cases we considered the connected correlators). Only the case $B=0$ is reported for clarity but also in the case of nonvanishing external magnetic field the behaviour of the correlators is qualitatively similar.

In the following we will need also the somehow less used correlator $C_{L L}(\mathbf{r}, T)$; a comparison of the behaviour of $C_{L L^{\dagger}}(r, T)$ and $\operatorname{Re} C_{L L}(r, T)$ for $T \simeq 200 \mathrm{MeV}$ is shown in Fig. 1, from which it is clear that the very large distance behavior of these two correlators is the same. In fact, the two correlators turn out to be substantially different only in the confined phase, where the $C_{L L}(\mathbf{r}, T)$ correlator is strongly suppressed also at short distances because of the confining properties of the medium ${ }^{1}$.

Under Euclidean-time reversal $\mathcal{R}: \tau \rightarrow-\tau$ the colormagnetic and color-electric gluon components, $A_{i}(\mathbf{x}, \tau)$ and $A_{4}(\mathbf{x}, \tau)$, are respectively even and odd. Since the Polyakov loop transforms under such a transformation as $\mathcal{R}: L \rightarrow L^{\dagger}$, the following combinations

$$
L_{M}=\frac{1}{2}\left(L+L^{\dagger}\right) \quad L_{E}=\frac{1}{2}\left(L-L^{\dagger}\right)
$$

receive contributions only from the magnetic and electric sectors respectively. These quantities can be further decomposed in eigenstates of the charge conjugation operator $\mathcal{C}$ : under this symmetry $\mathcal{C}: L \rightarrow L^{*}$ and we thus obtain

$$
L_{M^{ \pm}}=\frac{1}{2}\left(L_{M} \pm L_{M}^{*}\right) \quad L_{E^{ \pm}}=\frac{1}{2}\left(L_{E} \pm L_{E}^{*}\right)
$$

where the subscripts \pm indicates the $\mathcal{C}$ eigenvalues. Such a decomposition has been introduced in Ref. [44] and it has been recently used in some lattice computations (see e.g. [46, 47]).

\footnotetext{
${ }^{1}$ Actually, in the pure gauge theory, $C_{L L}(\mathbf{r}, T)$ vanishes exactly at all distances in the confined phase, because of the exact center symmetry.
} 
From the definitions in Eq. (5) it immediately follows that the magnetic odd and the electric even sectors are trivial, i.e.

$$
\operatorname{Tr} L_{M^{-}}=\operatorname{Tr} L_{E^{+}}=0,
$$

and that the following relations hold true

$$
\begin{aligned}
& \operatorname{Tr} L_{M^{+}}=\operatorname{Re} \operatorname{Tr} L \\
& \operatorname{Tr} L_{E^{-}}=i \operatorname{Im} \operatorname{Tr} L .
\end{aligned}
$$

Using the previous relations, the correlators needed to investigate the magnetic and electric sectors can be written in the form

$$
\begin{aligned}
& C_{M^{+}}(\mathbf{r}, T)=\left\langle\operatorname{Tr} L_{M^{+}}(\mathbf{0}) \operatorname{Tr} L_{M^{+}}(\mathbf{r})\right\rangle-|\langle\operatorname{Tr} L\rangle|^{2} \\
& C_{E^{-}}(\mathbf{r}, T)=-\left\langle\operatorname{Tr} L_{E^{-}}(\mathbf{0}) \operatorname{Tr} L_{E^{-}}(\mathbf{r})\right\rangle
\end{aligned}
$$

where the minus sign in the definition of $C_{E^{-}}$is conventional and in the electric case no disconnected term is present because of the symmetry under charge conjugation. It is convenient to rewrite the previous correlators in term of $C_{L L}$ and $C_{L L^{\dagger}}$ and the final result is

$$
\begin{aligned}
C_{M^{+}} & =+\frac{1}{2} \operatorname{Re}\left[C_{L L}+C_{L L^{\dagger}}\right]-|\langle\operatorname{Tr} L\rangle|^{2} \\
C_{E^{-}} & =-\frac{1}{2} \operatorname{Re}\left[C_{L L}-C_{L L^{\dagger}}\right] .
\end{aligned}
$$

Notice that the mixed electric-magnetic correlator

$$
\left\langle\operatorname{Tr} L_{M^{+}}(\mathbf{0}) \operatorname{Tr} L_{E^{-}}(\mathbf{r})\right\rangle \propto\langle\operatorname{Re} \operatorname{Tr} L(\mathbf{0}) \operatorname{Im} \operatorname{Tr} L(\mathbf{r})\rangle
$$

is zero by charge conjugation invariance, meaning that the real and imaginary parts of the Polyakov loop fluctuate independently.

The electric and magnetic screening masses can now be extracted from the large distance behaviour of the correlators in Eqs. (9). In particular, in the very high temperature regime, one expects [43, 44] these quantities to scale as

$$
\begin{aligned}
\left.C_{E^{-}}(\mathbf{r}, T)\right|_{r \rightarrow \infty} & \simeq \frac{e^{-m_{E}(T) r}}{r} \\
\left.C_{M^{+}}(\mathbf{r}, T)\right|_{r \rightarrow \infty} & \simeq \frac{e^{-m_{M}(T) r}}{r}
\end{aligned}
$$

where $r=|\mathbf{r}|$ and $m_{E}(T)$ and $m_{M}(T)$ are the electric and magnetic screening masses respectively. In the subsequent analysis, we will use these expressions to extract the screening masses from the correlators computed on the lattice. In the presence of an external magnetic field, we obviously have to take into account the explicit breaking of the rotational symmetry: correlators taken along directions parallel or orthogonal to the magnetic field cannot be averaged, and two a priori different electric screening masses and magnetic screening masses have to be defined.

\section{B. Numerical setup}

In this work we have adopted a discretization of $N_{f}=$ $2+1$ QCD based on the Symanzik tree-level improved gauge action and the stout smeared rooted staggered action for the fermionic sector. The partition function in the presence of a magnetic background $B$ is written as

$$
Z(B)=\int \mathcal{D} U e^{-\mathcal{S}_{Y M}} \prod_{f=u, d, s} \operatorname{det}\left(D_{\mathrm{st}}^{f}[B]\right)^{1 / 4},
$$

where $\mathcal{D} U$ is the functional integration over the $S U(3)$ gauge link variables, $\mathcal{S}_{Y M}$ stands for the tree-level improved action [48, 49]:

$$
\mathcal{S}_{Y M}=-\frac{\beta}{3} \sum_{i, \mu \neq \nu}\left(\frac{5}{6} W_{i ; \mu \nu}^{1 \times 1}-\frac{1}{12} W_{i ; \mu \nu}^{1 \times 2}\right),
$$

where $W_{i ; \mu \nu}^{1 \times 1}$ and $W_{i ; \mu \nu}^{1 \times 2}$ denote, respectively, the real part of the trace of $1 \times 1$ and $1 \times 2$ loops. Finally, the staggered fermion matrix

$$
\begin{aligned}
\left(D_{\mathrm{st}}^{f}\right)_{i, j}= & a m_{f} \delta_{i, j}+\sum_{\nu=1}^{4} \frac{\eta_{i ; \nu}}{2}\left(u_{i ; \nu}^{f} U_{i ; \nu}^{(2)} \delta_{i, j-\hat{\nu}}\right. \\
& \left.-u_{i-\hat{\nu} ; \nu}^{f *} U_{i-\hat{\nu} ; \nu}^{(2) \dagger} \delta_{i, j+\hat{\nu}}\right)
\end{aligned}
$$

is written in terms of two times stout-smeared gauge links $U_{i ; \mu}^{(2)}$ [50], with an isotropic smearing parameter $\rho=0.15$, and the $U(1)$ parallel transporters $u_{i ; \mu}^{f}$, which takes the presence of the external electromagnetic field into account; in both cases the latin indices denote the position in the lattice and the greek indices denote the direction of the link.

For a constant and uniform magnetic background directed along the $\hat{z}$ direction, a possible choice of the $U(1)$ phases is ( $q_{f}$ is the fermion charge)

$$
u_{i ; y}^{f}=e^{i a^{2} q_{f} B_{z} i_{x}},\left.\quad u_{i ; x}^{f}\right|_{i_{x}=L_{x}}=e^{-i a^{2} q_{f} L_{x} B_{z} i_{y}},
$$

while all the other $U(1)$ link variables are set to 1 . For this choice to actually describe a uniform magnetic field on the lattice with periodic boundary conditions, it is necessary for the value of $B_{z}$ to satisfy the following quantization condition 51 53.

$$
e B_{z}=6 \pi b_{z} /\left(a^{2} N_{x} N_{y}\right) ; \quad b_{z} \in \mathbb{Z} .
$$

In our numerical simulations, we used for the bare parameters the values $\beta=3.85, m_{s} / m_{l}=28.15$ and $a m_{s}=0.0394$, which correspond [54 56] to a lattice spacing $a \simeq 0.0989 \mathrm{fm}$ and to physical values of the pion and strange quark masses (isospin breaking is neglected). We performed simulations on $48^{3} \times N_{t}$ lattices, with $N_{t}=6,8,10$, corresponding to a fixed spatial size of around $5 \mathrm{fm}$ and to temperatures $T \simeq$ $330 \mathrm{MeV}, 250 \mathrm{MeV}, 200 \mathrm{MeV}$. Polyakov loop correlators 
have been measured on a set of around $5 \times 10^{3}$ configurations for each temperature (with measures separated by 5 molecular dynamics trajectories) and, to reduce the statistical noise, a single step of HYP smearing [57] has been applied to the temporal links, with the parameters of the HYP2-action, see Ref. [58]. Correlators have been extracted for generic orientations (i.e. not just along the lattice axes) at $B=0$, while in the presence of the background field we have considered separately correlators along the $z$ axis (i.e. parallel to $B$ ) and in the whole $x y$ plane (i.e. orthogonal to $B$ ), which in the following will be denoted respectively by $Z$ and $X Y$. Note that, since Polyakov loops renormalize multiplicatively and no further distance-dependent renormalization enters the correlator, the screening masses defined by Eq. (12) do not need any renormalization.

\section{RESULTS}

In Fig. 2, we show an example of the electric and magnetic correlators for $T \simeq 200 \mathrm{MeV}$ at $e|B|=0$ and $e|B| \simeq 1.3 \mathrm{GeV}^{2}$. At zero magnetic field all possible orientations are displayed, while for non-zero field only orientations perpendicular or parallel to the magnetic field are considered. For this reason, the three curves in each panel have different number of points.

The points at zero magnetic field approximately lay a single curve, indicating that the lattice violations of rotational invariance are small. This holds true also for correlators defined in the plane perpendicular to the magnetic field, indicating that the residual $O(2)$ subgroup of $O(3)$ left unbroken by the presence of $\mathbf{B}$ is well realized on the lattice for these correlator, and in short, that lattice artefacts are small.

The external magnetic field is expected to modify the correlators both by changing the screening masses and by inducing anisotropies in the correlators. Fig. 2] shows that both the electric and the magnetic correlators approach zero faster when an external magnetic field is present, a fact that implies that the screening masses are increasing functions of $B$. The anisotropy of the correlators is in general not easy to observe in the high temperature regime (see also [21] ), since correlators decay very quickly and it is possible to estimate them with enough relative accuracy only for a short distance; nevertheless, for the relatively low $T$ and high $B$ case shown in Fig. 2, the anisotropy is present and more pronounced in the magnetic correlator (whose signal is larger than the electric one).

In order to determine the screening masses from the correlators, numerical data have been fitted with the functional form in Eq. (12), adopting a bootstrap approach to propagate the correlations between data. Several fit intervals have been investigated in order to assess the stability and reliability of the results and to estimate the systematic uncertainties associated with the fitting procedure. In Table I we report the numerical values
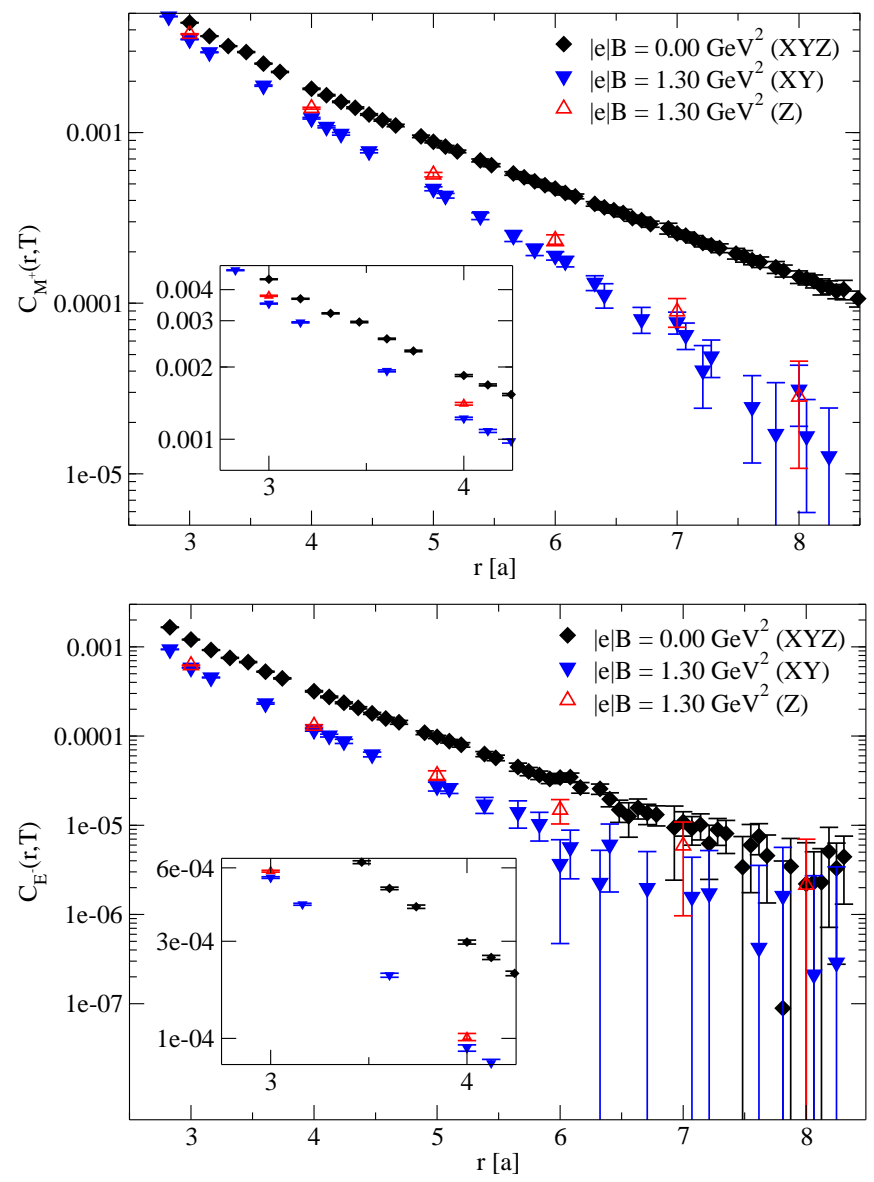

FIG. 2: Magnetic $C_{M^{+}}(r, T)$ (upper) and electric $C_{E^{-}}(r, T)$ (lower) correlators computed at $T \simeq 200 \mathrm{MeV}$ and with a magnetic field $|e| B \simeq 1.3 \mathrm{GeV}^{2}$. Correlators corresponding to separations parallel or orthogonal to the external magnetic field are denoted respectively by $Z$ and $X Y$. For comparison the results obtained for $e|B|=0$ are also displayed.

obtained and in Figs. 3, 4 we show a graphical representation of their behavior as a function of $|e| B$ and $T$.

At vanishing magnetic field we reproduced the known behavior of $m_{E}$ and $m_{M}$ : the electric screening mass is larger than the magnetic one and the ratios $m_{M} / T$ and $m_{E} / T$ are remarkably insensitive to the value of the temperature, something that a priori would have been expected to hold only at much higher temperatures. Our results are in good agreement with the corresponding temperatures and lattice spacing data presented in Ref. [47], where the same discretization was used and to which we refer for an in depth discussion of the $B=0$ case.

As previously anticipated from Fig. 2, the effect of the magnetic field is to increase both the magnetic and the electric screening mass, as visible in Fig. 3. In both cases the growth is roughly linear in the magnetic field and with similar slopes. Indeed the ratio $m_{E}(T, B) / m_{M}(T, B)$ turns out to be independent of both the magnetic field intensity and the temperature, as shown in Fig. [5. In particular this means that that in 


\begin{tabular}{c|c|c|c|c|c}
\hline \hline$T[\mathrm{MeV}]$ & $|e| B\left[\mathrm{GeV}^{2}\right]$ & $m_{M}^{X Y} / T$ & $m_{M}^{Z} / T$ & $m_{E}^{X Y} / T$ & $m_{E}^{Z} / T$ \\
\hline 330 & 0.00 & $5.12(18)$ & $5.12(18)$ & $9.07(56)$ & $9.07(56)$ \\
$"$ & 0.26 & $5.16(17)$ & $4.92(16)$ & $8.71(61)$ & $9.50(60)$ \\
$"$ & 0.52 & $5.20(19)$ & $5.01(17)$ & $9.36(74)$ & $9.71(67)$ \\
$"$ & 0.78 & $5.17(19)$ & $5.16(16)$ & $9.15(56)$ & $9.51(66)$ \\
$"$ & 1.04 & $5.41(19)$ & $5.16(15)$ & $9.36(49)$ & $8.46(76)$ \\
$"$ & 1.30 & $5.77(19)$ & $5.32(17)$ & $10.39(60)$ & $9.69(47)$ \\
\hline 250 & 0.00 & $4.70(17)$ & $4.70(17)$ & $9.54(58)$ & $9.54(58)$ \\
$"$ & 0.26 & $5.11(16)$ & $5.11(17)$ & $9.47(67)$ & $9.33(68)$ \\
$"$ & 0.52 & $5.12(18)$ & $5.22(21)$ & $9.64(57)$ & $9.66(60)$ \\
$"$ & 0.78 & $5.60(16)$ & $5.39(18)$ & $9.85(42)$ & $9.45(49)$ \\
$"$ & 1.04 & $5.98(16)$ & $5.60(18)$ & $10.20(64)$ & $9.57(60)$ \\
$"$ & 1.30 & $6.67(19)$ & $5.84(19)$ & $10.78(72)$ & $10.59(73)$ \\
\hline 200 & 0.00 & $4.80(22)$ & $4.80(22)$ & $9.65(35)$ & $9.65(35)$ \\
$"$ & 0.26 & $5.61(21)$ & $5.59(20)$ & $9.19(54)$ & $9.84(68)$ \\
$"$ & 0.52 & $6.14(24)$ & $5.61(26)$ & $10.54(43)$ & $9.71(60)$ \\
$"$ & 0.78 & $6.59(18)$ & $6.30(17)$ & $11.55(52)$ & $11.88(61)$ \\
$"$ & 1.04 & $7.18(21)$ & $6.55(24)$ & $13.09(76)$ & $12.57(74)$ \\
$"$ & 1.30 & $7.70(25)$ & $6.93(23)$ & $12.84(55)$ & $12.52(43)$ \\
\hline \hline
\end{tabular}

TABLE I: Screening masses obtained at three different temperatures and for several magnetic field intensities. Data at $|e| B=0$ have been obtained by averaging over all the spatial directions.

the regime studied in this work the external magnetic field does not change the usual $m_{M}<m_{E}$ hierarchy.

In the case of $m_{M}$ an anisotropy is observed, as could have been guessed by Fig. 2, with the screening mass relative to the directions orthogonal to the external field being larger than the one in the direction parallel to the field. In the case of the electric screening mass no such an anisotropy is observed, however this could be due to the fact that data for $m_{E}$ have larger relative error with respect to the ones for $m_{B}$. The different accuracy of these estimates is a consequence of the relation $m_{E}>m_{M}$ : magnetic and electric correlators have similar statistical (absolute) errors, but the extraction of $m_{E}$ is made difficult by the rapid decrease of the electric correlator.

We now try to determine a functional form that well describes the $T$ and $B$ dependence of the screening masses. From Fig. 3 and Fig. 4 it follows that the main properties of the screening masses, at least in the explored range of temperatures and magnetic field intensities, are:

i) at $B=0$ the ratios $m_{E} / T$ and $m_{M} / T$ are independent of $T$;

ii) for large magnetic field the screening masses grow linearly with $B$.

To these properties, it is reasonable to add the requirement that the screening masses be analytic functions of the magnetic field $\mathbf{B}$. From that it follows that, in the limit of small magnetic field intensity, the behaviors of $m_{E}$ and $m_{M}$ have to be quadratic in $|e| B$. In the high temperature phase it also seems reasonable to assume the only relevant dimensional parameters to be the temperature and the magnetic field intensity.

Before going on we can explicitly check, in a model independent way, that $B$ and $T$ are the only dimensional
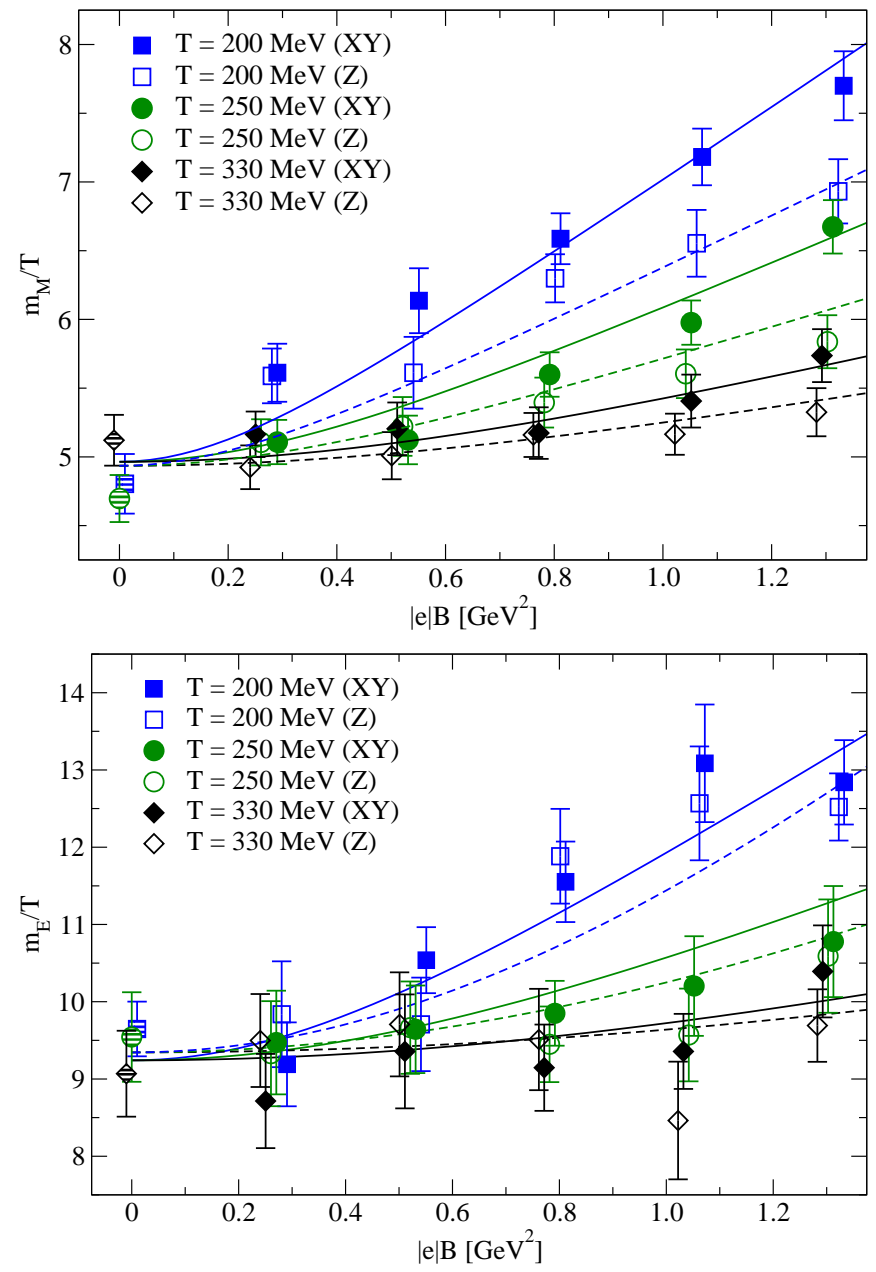

FIG. 3: Behavior of the ratios $m_{M} / T$ (upper) and $m_{E} / T$ (lower) as a function of the external field directed along $\hat{z}$. Data points (slightly shifted on the horizontal axis to improve readability) are shown together with the best fit curves obtained by using the model in Eq. (17).

quantities that matter to describe the behavior of the screening masses, by showing that the ratios $m_{E} / T$ and $m_{M} / T$ depend only on the dimensionless combination $B / T^{2}$. This is indeed the case, as can be appreciated from Fig. 6, where the $B^{2}$ behaviour for small values of the magnetic field is also somehow more clearly visible than in Figs 3,4,

A simple ansatz that satisfies all the previous properties is

$$
\frac{m_{E}^{d}}{T}=a_{E}^{d}\left[1+c_{1 ; E}^{d} \frac{|e| B}{T^{2}} \operatorname{atan}\left(\frac{c_{2 ; E}^{d}}{c_{1 ; E}^{d}} \frac{|e| B}{T^{2}}\right)\right],
$$

where $d$ denotes the spatial direction (i.e. $d=X Y$ or $d=Z$ ) and an analogous expression can be used for the magnetic screening mass. This functional form is analogous to the one used in Ref. [59] for the case of the dependence of the chiral condensate on the magnetic field. 

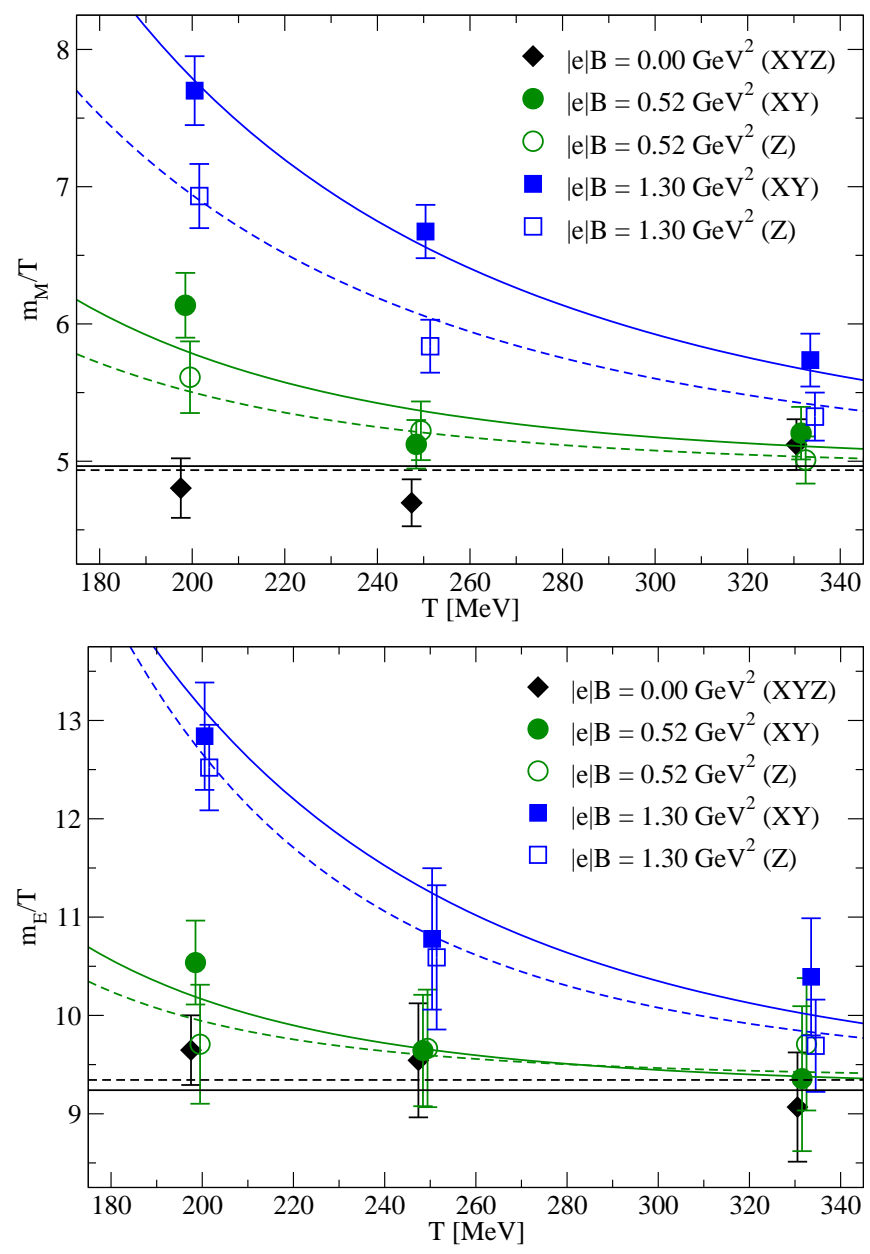

FIG. 4: Behavior of the ratios $m_{M} / T$ (upper) and $m_{E} / T$ (lower) as a function of the temperature. Data points (sligthly shifted on the horizontal axis to improve readability) are shown together with the best fit curves obtained by using the model in Eq. (17).

The three parameters that enter the ansatz in Eq. (17) have simple interpretations: $a_{E}$ is the $B=0$ value of the ratio $m_{E} / T$ (that is known to be $T$-independent), $c_{1, E}$ is related to the slope of $m_{E}$ as a function of $B$ at fixed temperature for large magnetic field intensities, while $c_{2, E}$ is associated with the quadratic small- $B$ behavior of $m_{E}$.

The best fit values for the parameters entering Eq. (17) for both $m_{E}$ and $m_{M}$ are reported in Table I. While for the magnetic screening masses reasonable values of the $\chi^{2}$ test are obtained, for the case of the electric masses the values of $\chi^{2} /$ d.o.f. are somehow small, indicating that we are using more parameters than needed to fit the data at the current level of statistical accuracy. Indeed a simple linear dependence on $|e| B$ is sufficient to describe the data for the electric masses, however this is just a consequence of the large error bars on the electric correlator.

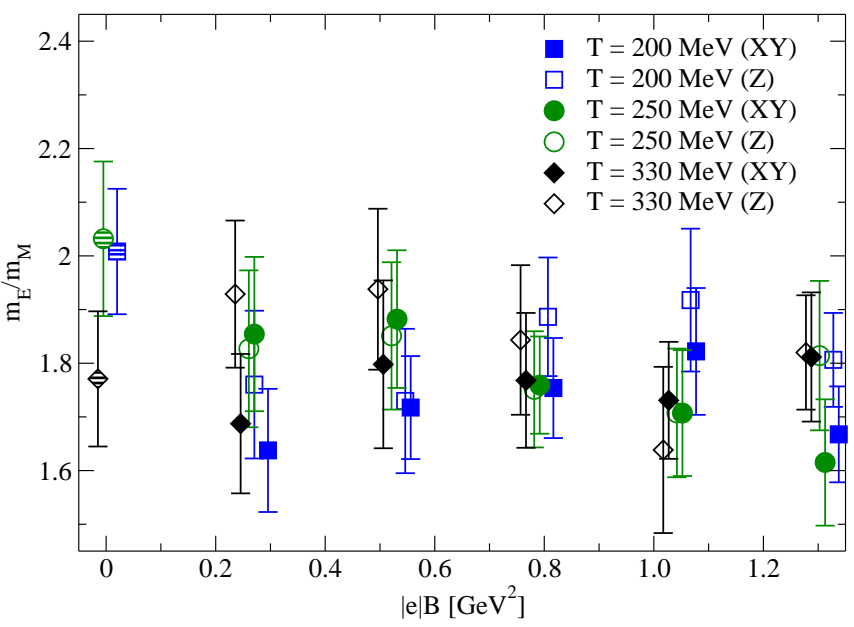

FIG. 5: Determinations of the ratio $m_{E} / m_{M}$ for different temperatures and external field intensity.

\begin{tabular}{l|c|c|c|c}
\hline \hline & $a$ & $c_{1}$ & $c_{2}$ & $\chi^{2} /$ d.o.f \\
\hline$m_{M}^{X Y}$ & $4.964(82)$ & $0.137(19) \times 10^{-1}$ & $0.141(55) \times 10^{-2}$ & 1.06 \\
$m_{M}^{Z}$ & $4.935(79)$ & $0.099(20) \times 10^{-1}$ & $0.094(49) \times 10^{-2}$ & 1.10 \\
$m_{E}^{X Y}$ & $9.24(21)$ & $0.120(47) \times 10^{-1}$ & $0.069(38) \times 10^{-2}$ & 0.63 \\
$m_{E}^{Z}$ & $9.34(20)$ & $0.17(28) \times 10^{-1}$ & $0.039(21) \times 10^{-2}$ & 0.85 \\
\hline \hline
\end{tabular}

TABLE II: Best fit values for the parameters entering the functional form in Eq. (17); in all cases d.o.f. $=16$.

\section{CONCLUSIONS}

In this study we have investigated the effects of a magnetic background on color-screening phenomena taking place in the Quark-Gluon Plasma. To that purpose, we have measured Polyakov loop correlators for various temperatures, up to $T \simeq 330 \mathrm{MeV}$, and uniform magnetic fields going up to $|e| B \simeq 1.3 \mathrm{GeV}^{2}$. Our results have been obtained at a single value of the lattice spacing, $a \sim 0.0989 \mathrm{fm}$, and a refinement of the analysis, aimed at a continuum limit extrapolation, should be performed in the future.

We have shown that the magnetic field induces an increase of both the magnetic and the electric screening masses and, to some extent, also the appearance of an anisotropy in Polyakov loop correlators. The masses increase linearly with the magnetic field for moderate or large $B$ values (i.e. for $|e| B \gtrsim 0.2 \mathrm{GeV}^{2}$ ) and a reasonable ansatz can be given to describe the connection of this regime with the expected quadratic behaviour for small values of $B$, in which both screening masses are proportional to $T$ and to a function of $B / T^{2}$. Indeed the influence of the magnetic field is stronger at lower temperatures and asymptotically vanishes in the large $T$ limit. On the other hand, the ratio of the magnetic to electric screening masses turns to be independent of $B$, within errors, with the magnetic screening mass always being smaller than the electric one. 

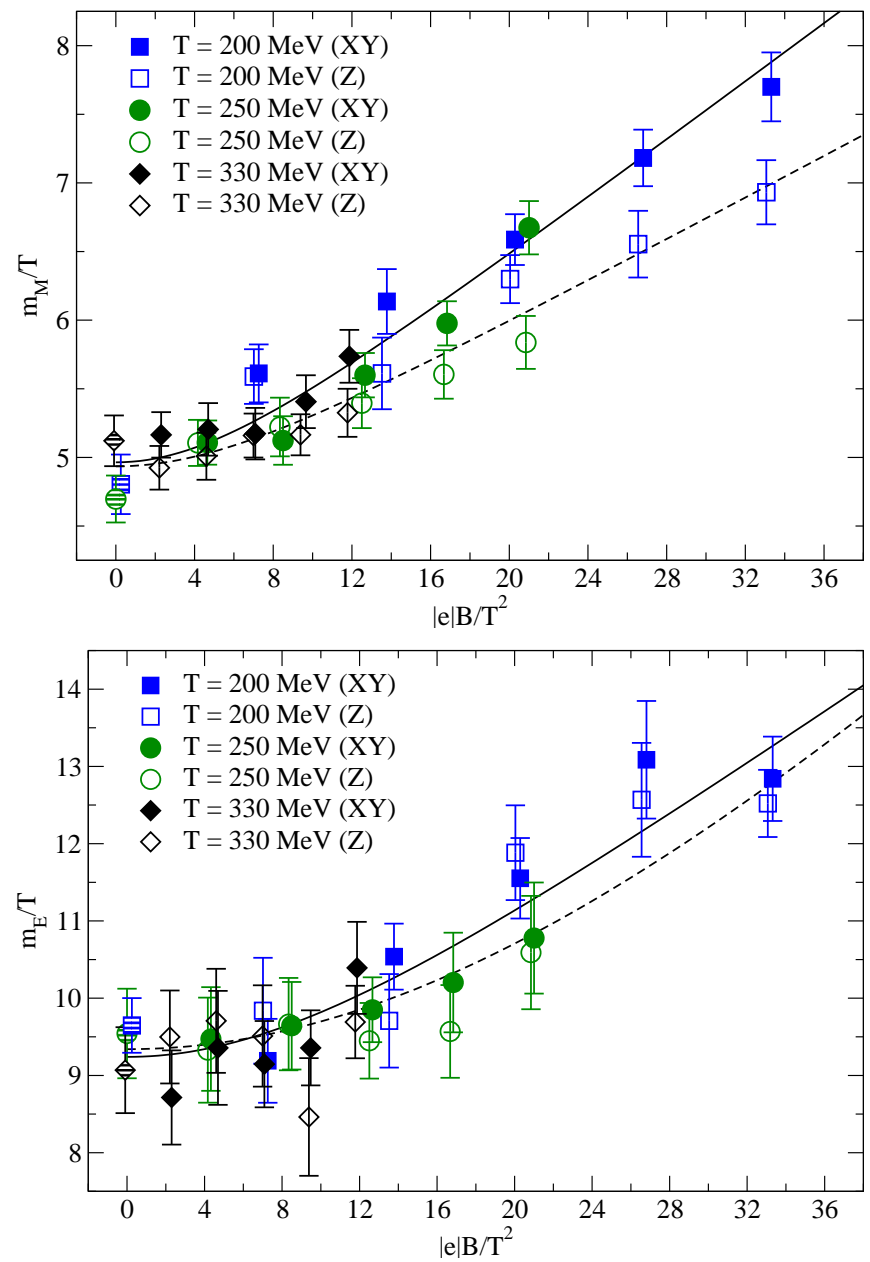

FIG. 6: Behavior of the ratios $m_{M} / T$ (upper) and $m_{E} / T$ (lower) as a function of $B / T^{2}$. Data points are shown together with the best fit curves obtained by using the model in Eq. (17).

The observed increase of the screening masses as a function of $B$ is in qualitative agreement with perturbative computations $60-62$ and with the behavior already observed below $T_{c}$ : the magnetic background tends to suppress the confining properties of the thermal medium below $T_{c}$, and to enhance the screening of color interactions above $T_{c}$. In both cases, one can interpret the effect in terms of the decrease of the pesudo-critical temperature $T_{c}$ as a function of $B$ [33], so that in the low temperature phase one approaches deconfinement as $B$ increases, while at high temperature one gets farther from the transition and color screening gets stronger by increasing $B$. Following this line of reasoning, no particular critical behavior is expected in the high temperature phase when approaching the large magnetic field limit, since the system will just become more deconfined (i.e. color interactions will be more and more screened); this is in contrast to what happens below $T_{c}$ or to what might happen even at $T=0$ [21, 63].

The increasing of the screening masses induced by the presence of an external magnetic field could in principle lead to a stronger suppression of heavy quark bound states in peripheral heavy-ion collisions and, more specifically, to a direct relation between suppression and centrality. However, following the original argument presented in Ref. 1], an in deep discussion should consider also the modifications of the radius of the heavy quarks bound states as a function of $B$, since it is the reduction of the screening length with respect to such radius which brings to the actual suppression; a direct computation of quarkonia spectral functions in the presence of magnetic background is surely something that should be addressed by future lattice studies. Moreover, in order to assess the relevance of our results to heavy ion phenomenology, one should first of all know to which extent the magnetic field produced in non-central heavy ion collisions survives the thermalization process, not to mention all the other dynamical processes that make it difficult to safely predict the fate of heavy quark bound states even in the absence of external magnetic field.

\section{Acknowledgments}

We acknowledge PRACE for awarding us access to resource FERMI based in Italy at CINECA, under project Pra09-2400 - SISMAF.
[1] T. Matsui and H. Satz, Phys. Lett. B 178, 416 (1986).

[2] A. Andronic et al., Eur. Phys. J. C 76, no. 3, 107 (2016) arXiv:1506.03981 [nucl-ex]].

[3] T. Vachaspati, Phys. Lett. B 265, 258 (1991).

[4] D. Grasso and H. R. Rubinstein, Phys. Rept. 348, 163 (2001) astro-ph/0009061.

[5] V. Skokov, A. Y. Illarionov and V. Toneev, Int. J. Mod. Phys. A 24, 5925 (2009) arXiv:0907.1396 [nucl-th]].

[6] V. Voronyuk, V. D. Toneev, W. Cassing, E. L. Bratkovskaya, V. P. Konchakovski and S. A. Voloshin, Phys. Rev. C 83, 054911 (2011) arXiv:1103.4239 [nucl-th]].
[7] A. Bzdak and V. Skokov, Phys. Lett. B 710, 171 (2012) arXiv:1111.1949 [hep-ph]].

[8] W. -T. Deng and X. -G. Huang, Phys. Rev. C 85, 044907 (2012) arXiv:1201.5108 [nucl-th]].

[9] K. Tuchin, Adv. High Energy Phys. 2013, 490495 (2013) arXiv:1301.0099.

[10] R. Holliday and K. Tuchin, arXiv:1604.04572 [hep-ph].

[11] R. C. Duncan and C. Thompson, Astrophys. J. 392, L9 (1992).

[12] D. E. Kharzeev, K. Landsteiner, A. Schmitt and H. U. Yee, Lect. Notes Phys. 871, 1 (2013).

[13] V. A. Miransky and I. A. Shovkovy, Phys. Rept. 576, 1 
(2015) arXiv:1503.00732 [hep-ph]].

[14] V. A. Miransky and I. A. Shovkovy, Phys. Rev. D 66, 045006 (2002) hep-ph/0205348.

[15] M. N. Chernodub, Mod. Phys. Lett. A 29, 1450162 (2014).

[16] R. Rougemont, R. Critelli and J. Noronha, Phys. Rev. D 91, 066001 (2015) arXiv:1409.0556 [hep-th]].

[17] E. J. Ferrer, V. de la Incera and X. J. Wen, Phys. Rev. D 91, 054006 (2015) arXiv:1407.3503 [nucl-th]].

[18] Y. A. Simonov and M. A. Trusov, Phys. Lett. B 747, 48 (2015) arXiv:1503.08531 [hep-ph]].

[19] M. D'Elia, E. Meggiolaro, M. Mesiti and F. Negro, Phys. Rev. D 93, 054017 (2016) doi:10.1103/PhysRevD.93.054017 arXiv:1510.07012 [hep-lat]].

[20] C. Bonati, M. D'Elia, M. Mariti, M. Mesiti, F. Negro and F. Sanfilippo, Phys. Rev. D 89, 114502 (2014) arXiv:1403.6094 [hep-lat]].

[21] C. Bonati, M. D'Elia, M. Mariti, M. Mesiti, F. Negro, A. Rucci and F. Sanfilippo, Phys. Rev. D 94, 094007 (2016) arXiv:1607.08160 [hep-lat]].

[22] J. Alford and M. Strickland, Phys. Rev. D 88, 105017 (2013) arXiv:1309.3003 [hep-ph]].

[23] D. Dudal and T. G. Mertens, Phys. Rev. D 91, 086002 (2015) arXiv:1410.3297 [hep-th]].

[24] S. Cho, K. Hattori, S. H. Lee, K. Morita and S. Ozaki, Phys. Rev. D 91, 045025 (2015) arXiv:1411.7675 [hep$\mathrm{ph}]$.

[25] H. Taya, Phys. Rev. D 92, $014038 \quad$ (2015) arXiv:1412.6877 [hep-ph]].

[26] C. Bonati, M. D'Elia and A. Rucci, Phys. Rev. D 92, 054014 (2015) arXiv:1506.07890 [hep-ph]].

[27] K. Hattori, T. Kojo and N. Su, Nucl. Phys. A 951, 1 (2016) arXiv:1512.07361 [hep-ph]].

[28] K. Suzuki and T. Yoshida, Phys. Rev. D 93, 051502 (2016) arXiv:1601.02178 [hep-ph]]; arXiv:1607.04935 [hep-ph].

[29] X. Guo, S. Shi, N. Xu, Z. Xu and P. Zhuang, Phys. Lett. B 751, 215 (2015) arXiv:1502.04407 [hep-ph]].

[30] K. Fukushima, K. Hattori, H. U. Yee and Y. Yin, Phys. Rev. D 93, 074028 (2016) arXiv:1512.03689 [hep-ph]].

[31] P. Gubler, K. Hattori, S. H. Lee, M. Oka, S. Ozaki and K. Suzuki, Phys. Rev. D 93, 054026 (2016) arXiv:1512.08864 [hep-ph]].

[32] M. Hasan, B. Chatterjee and B. K. Patra, arXiv:1703.10508 [hep-ph].

[33] G. S. Bali, F. Bruckmann, G. Endrodi, Z. Fodor, S. D. Katz, S. Krieg, A. Schafer and K. K. Szabo, JHEP 1202, 044 (2012) arXiv:1111.4956 [hep-lat]].

[34] F. Bruckmann, G. Endrodi and T. G. Kovacs, JHEP 1304, 112 (2013) arXiv:1303.3972 [hep-lat]].

[35] E.-M. Ilgenfritz, M. Muller-Preussker, B. Petersson and A. Schreiber, Phys. Rev. D 89, 054512 (2014) arXiv:1310.7876 [hep-lat]].

[36] D. J. Gross, R. D. Pisarski and L. G. Yaffe, Rev. Mod. Phys. 53, 43 (1981).

[37] J. I. Kapusta, C. Gale "Finite-Temperature Field Theory. Principles and Applications" Cambridge University Press
(2006).

[38] M. Laine, A. Vuorinen "Basics of thermal field theory - a tutorial on perturbative computations" Lect. Notes Phys. 925 (2016) pp.1-281 [arXiv:1701.01554 [hep-ph]].

[39] S. Nadkarni, Phys. Rev. D 333738 (1986)

[40] A. K. Rebhan, Phys. Rev. D 48, R3967 (1993) hep-ph/9308232.

[41] A. K. Rebhan, Nucl. Phys. B 430, 319 (1994) hep-ph/9408262.

[42] E. Braaten and A. Nieto, Phys. Rev. Lett. 73, 2402 (1994) doi:10.1103/PhysRevLett.73.2402 hep-ph/9408273.

[43] E. Braaten and A. Nieto, Phys. Rev. Lett. 74, 3530 (1995) hep-ph/9410218.

[44] P. B. Arnold and L. G. Yaffe, Phys. Rev. D 52, 7208 (1995) hep-ph/9508280.

[45] L. D. McLerran and B. Svetitsky, Phys. Rev. D 24, 450 (1981).

[46] Y. Maezawa et al. [WHOT-QCD Collaboration], Phys. Rev. D 81, 091501 (2010) arXiv:1003.1361 [hep-lat]].

[47] S. Borsnyi, Z. Fodor, S. D. Katz, A. Psztor, K. K. Szab and C. Trk, JHEP 1504, 138 (2015) arXiv:1501.02173 [hep-lat]].

[48] P. Weisz, Nucl. Phys. B 212, 1 (1983).

[49] G. Curci, P. Menotti and G. Paffuti, Phys. Lett. B 130, 205 (1983) [Erratum-ibid. B 135, 516 (1984)].

[50] C. Morningstar and M. J. Peardon, Phys. Rev. D 69, 054501 (2004) hep-lat/0311018.

[51] G. 't Hooft, Nucl. Phys. B 153, 141 (1979).

[52] P. H. Damgaard and U. M. Heller, Nucl. Phys. B 309, 625 (1988).

[53] M. H. Al-Hashimi and U. J. Wiese, Ann. Phys. 324, 343 (2009) arXiv:0807.0630 [quant-ph]].

[54] Y. Aoki, S. Borsanyi, S. Durr, Z. Fodor, S. D. Katz, S. Krieg and K. K. Szabo, JHEP 0906, 088 (2009) arXiv:0903.4155 [hep-lat]].

[55] S. Borsanyi, G. Endrodi, Z. Fodor, A. Jakovac, S. D. Katz, S. Krieg, C. Ratti and K. K. Szabo, JHEP 1011, 077 (2010) arXiv:1007.2580 [hep-lat]];

[56] S. Borsanyi, Z. Fodor, C. Hoelbling, S. D. Katz, S. Krieg and K. K. Szabo, Phys. Lett. B 730, 99 (2014) arXiv:1309.5258 [hep-lat]].

[57] A. Hasenfratz and F. Knechtli, Phys. Rev. D 64, 034504 (2001) hep-lat/0103029.

[58] M. Della Morte, A. Shindler and R. Sommer, JHEP 0508, 051 (2005) hep-lat/0506008.

[59] M. D'Elia and F. Negro, Phys. Rev. D 83, 114028 (2011) arXiv:1103.2080 [hep-lat]].

[60] J. Alexandre, Phys. Rev. D 63, 073010 (2001) hep-th/0009204.

[61] A. Bandyopadhyay, N. Haque and M. G. Mustafa, arXiv:1702.02875 [hep-ph].

[62] A. Bandyopadhyay, C. A. Islam and M. G. Mustafa, Phys. Rev. D 94, no. 11, 114034 (2016) arXiv:1602.06769 [hep-ph]].

[63] G. Endrodi, JHEP 1507, 173 (2015) arXiv:1504.08280 [hep-lat]]. 